\title{
Contribuições do enfermeiro na educação e gerenciamento do cuidado em tratamento conservador em nefrologia: revisão integrativa
}

\author{
Nurse's contributions in education and care management in conservative treatment in nephrology: \\ integrative review \\ Contribuciones de las enfermeras a la educación y gestión de los cuidados en el tratamiento \\ conservador en nefrología: revisión integradora
}

Rafael Abrantes de Lima

ORCID: https://orcid.org/0000-0002-3843-7297 Universidade Federal do Estado do Rio de Janeiro, Brasil

E-mail: rafael.abrantes83@gmail.com

Tamires Zêba Guimarães

ORCID: https://orcid.org/0000-0001-5832-7696 Universidade Federal do Estado do Rio de Janeiro, Brasil E-mail: mireszg@gmail.com

Daniela de Oliveira Matias

ORCID: https://orcid.org/0000-0003-4177-6799 Universidade Federal do Estado do Rio de Janeiro, Brasil E-mail: danielamatiasenf@hotmail.com

Letícia Lima Borges

ORCID: https://orcid.org/0000-0002-2366-8092 Universidade Federal do Estado do Rio de Janeiro, Brasil E-mail: lima.leticia.borges@gmail.com

Vanessa Peres Cardoso Pimentel

ORCID: https://orcid.org/0000-0002-0391-281X Universidade Federal do Estado do Rio de Janeiro, Brasil E-mail: nessaserep@hotmail.com

Izabella Andrade da Rocha

ORCID: https://orcid.org/0000-0002-9121-0972

Universidade do Estado do Rio de Janeiro, Brasil E-mail: Iza_belbella@hotmail.com

Gicélia Lombardo Pereira

ORCID: https://orcid.org/0000-0002-4032-2093 Universidade Federal do Estado do Rio de Janeiro, Brasil E-mail: gilombardo@ hotmail.com

Beatriz Gerbassi Costa Aguiar ORCID: https://orcid.org/0000-0001-6815-4354 Universidade Federal do Estado do Rio de Janeiro, Brasil E-mail: beatriz.costa@unirio.br

Brenda Maia do Nascimento

ORCID: https://orcid.org/0000-0002-3691-9401 Universidade Federal do Estado do Rio de Janeiro, Brasil E-mail: brendamaia1512@gmail.com

\begin{abstract}
Resumo
A Doença Renal Crônica é um problema de saúde pública mundial e tem como principais fatores a hipertensão arterial sistêmica e em segundo lugar a diabetes mellitus associados ou não a outros fatores como etilismo, tabagismo, envelhecimento populacional. O objetivo do estudo é identificar as evidências científicas sobre as contribuições dos enfermeiros na educação e no gerenciamento do cuidado de clientes nefropatas em tratamento conservador. Metodologia: trata-se de uma revisão integrativa, cujo método permite a análise de múltiplos estudos e, assim, a síntese, contribuindo para elaborar conclusões a respeito de um determinado tema. Resultados: a pesquisa foi composta por nove artigos, o ano de 2013 foi o que apresentou maior número de publicações com três artigos, quanto ao país de origem destaca-se o Brasil com oito artigos publicados e a Revista RENE foi a com maior número de artigos publicados. Destaca-se o papel fundamental do enfermeiro inserido nos ambulatórios de tratamento conservador, que articula com as demais categorias profissionais em busca de melhor condições e qualidade.
\end{abstract}


Evidencia-se a necessidade de conhecer a visão do paciente em relação a doença renal crônica, com o intuito de partilhar melhor as decisões a serem tomadas acerca de tratamento, rede de apoio e profissionais de saúde.

Palavras-chave: Cuidados de enfermagem; Tratamento conservador; Enfermagem; Insuficiência renal crônica; Educação em saúde.

\begin{abstract}
Chronic Kidney Disease is a global public health problem, and its main factors are systemic arterial hypertension and secondly, diabetes mellitus associated or not with other factors such as alcoholism, smoking, and population aging. The aim of this study is to identify the scientific evidence on nurses' contributions to education and to managing the care of nephropathic clients undergoing conservative treatment. Methodology: this is an integrative review, whose method allows the analysis of multiple studies and, thus, the synthesis, which contributes to draw conclusions on a given theme. Results: the research was composed of nine articles, 2013 was the year that presented the highest number of publications including three articles, as for the country of origin, Brazil stands out with eight articles published and Revista RENE was the journal with the highest number of articles published. The fundamental role of nurses inserted in conservative treatment outpatient facilities stands out, which articulates with the other professional categories in search of better conditions and quality. It highlights a need to know the patient's perspective in relation to chronic kidney disease, to better share the decisions to be made about treatment, support network and health professionals.
\end{abstract}

Keywords: Nursing care; Conservative treatment; Nursing; Renal insufficiency, chronic; Health education.

\begin{abstract}
Resumen
La Enfermedad Renal Crónica es un problema de salud pública global y sus principales factores son la hipertensión arterial sistémica y la diabetes mellitus en segundo lugar, asociadas o no a otros factores como el alcoholismo, el tabaquismo, y el envejecimiento de la población. El objetivo del estudio es identificar evidencias científicas sobre las contribuciones del enfermero en la educación y en el manejo de la atención de los pacientes con nefropatía en tratamiento conservador. Metodología: se trata de una revisión integradora, cuyo método permite el análisis de múltiples estudios y, por tanto, la síntesis, contribuyendo a sacar conclusiones sobre un tema determinado. Resultados: la investigación constó de nueve artículos. El año 2013 fue el de mayor número de publicaciones, con tres artículos. En cuanto al país de origen, se destaca Brasil con ocho artículos publicados. La Revista RENE fue la de mayor número de artículos publicados. Se destaca el papel fundamental del enfermero insertado en las clínicas de tratamiento conservador, que se articula con otras categorías profesionales en busca de mejores condiciones y calidad. Se resalta la necesidad de conocer la visión del paciente sobre la enfermedad renal crónica, con el fin de compartir mejor la toma de decisiones respecto al tratamiento, la red de apoyo, y los profesionales de la salud.
\end{abstract}

Palabras clave: Atención de enfermería; Tratamiento conservador; Enfermería; Insuficiencia renal crónica; Educación para la salud.

\title{
1. Introdução
}

A doença renal crônica (DRC) está no rol de doenças crônicas não transmissíveis (DCNT), tendo sido responsável por mais de 36 milhões de mortes no mundo, o que equivale a 63\% de todas as mortes (World Health Organization, 2013). No Brasil, as DCNT foram responsáveis por 54,7\% mortes no país (Ministério da Saúde, 2020).

Dessa forma, a DRC tornou-se um problema de saúde pública mundial e tem como principais fatores a hipertensão arterial sistêmica (HAS) e a diabetes mellitus (DM), associados ou não a outros fatores como etilismo, tabagismo, envelhecimento populacional (Castro, 2019) (Neves et al., 2020).

Segundo Abreu e Moura (2018) a DRC é caracterizada como doença irreversível e progressiva em que um adulto em um período de três ou mais meses apresentarem taxa de filtração glomerular (TFG) com $\left\langle 60 \mathrm{ml} / \mathrm{min} / 1,73 \mathrm{~m}^{2}\right.$ ou com TFG > $60 \mathrm{ml} / \mathrm{min} / 1,73 \mathrm{~m}^{2}$, mas que apresente algum tipo de lesão estrutural nos néfrons, tornando-se, assim, incapaz de manter a homeostase.

Para estruturação do atendimento a esses pacientes, torna-se necessário após seu diagnóstico definido que ele seja classificado dentro da estratificação da DRC e que possui estreita relação com o desfecho do seu tratamento e que pode ser observado no Quadro 1. 
Quadro 1. Classificação da DRC. Ministério da Saúde, 2014ª .

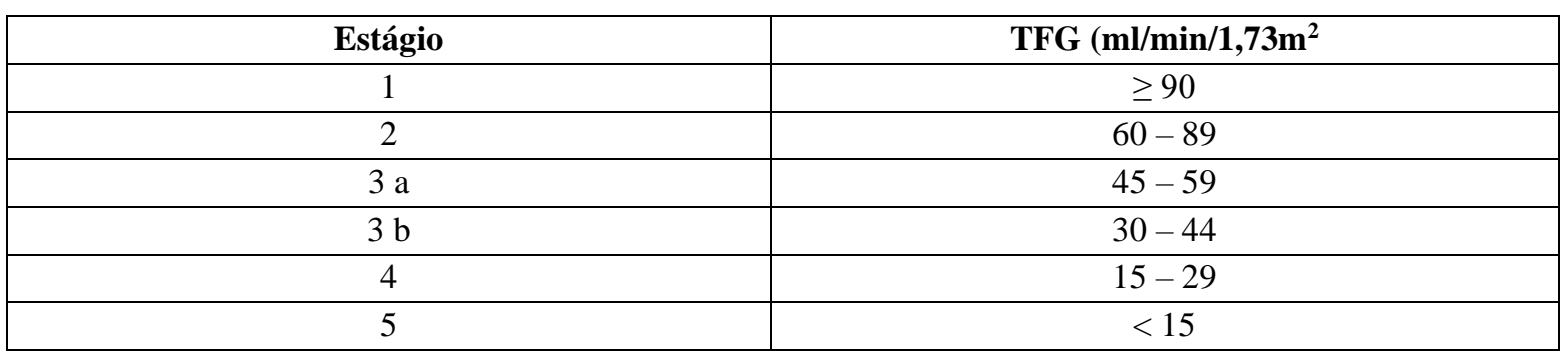

Fonte: Ministério da Saúde. (2014a).

O tratamento conservador consiste em controlar os fatores de risco que o paciente possui, a fim de retardar a progressão da doença e conservar a TFG por maior tempo possível. Os pacientes que acompanhados pelo tratamento conservador são aqueles que se enquadram entre os estágios 1 a 3 a. Tal qual, deve existir em consonância com adesão ao tratamento medicamentoso, equilíbrio na ingesta hídrica, acompanhamento dietético, mudança de hábitos de vida, mudanças no estilo de vida para que o tratamento seja adequado e cumpra seu papel no retardo da progressão da doença (Ministério da Saúde, 2014a).

Dentro desse contexto, o enfermeiro atua no desenvolvimento de medidas preventivas e educativas para que o cuidado seja de forma integral, sistematizado e individual às pessoas que passam a integrar o tratamento conservador e, assim, retarde sua inserção em alguma terapia renal substitutiva (TRS).

Por certo, o enfermeiro destaca-se como gerente do cuidado que busca a qualidade na assistência prestada através da articulação com o serviço em si e com outras categorias profissionais, mas sempre com foco no paciente (Bica et al., 2020).

O gerenciamento do cuidado exige planejamento e organização, caracterizando-se como um desafio ainda maior aliar a educação em saúde aos usuários. Segundo Siewert et al. (2017, p. 2) "É necessário buscar a complexidade no seu cotidiano, atendendo às demandas dos sujeitos e compreendendo-os como um ser singular e plural que, simultaneamente, representam a parte e o todo". O enfermeiro precisa ser um profissional com competência na dimensão física, emocional e espiritual, preparado a atender as mais diversas situações.

Desse modo, ações educativas são consideradas prioritárias nas políticas de prevenção, diagnóstico e tratamento precoce, que permitem desenvolver nos pacientes uma consciência crítica da causa dos problemas que os levaram até sua condição de DRC. Enfim, as mudanças nos métodos assistenciais que serão direcionadas as práticas educativas em saúde e são ainda primárias e têm visibilidade no cenário nacional (Lima et al., 2014).

O objetivo do estudo é identificar as evidências científicas sobre as contribuições dos enfermeiros na educação e no gerenciamento do cuidado de clientes nefropatas em tratamento conservador.

\section{Metodologia}

Trata-se de uma revisão integrativa, que não é só mais uma etapa em um processo de pesquisa, cujo método permite a análise de múltiplos estudos e, assim, a síntese, contribuindo para elaborar conclusões a respeito de um determinado tema. Foi desenvolvida em seis etapas: formulação da questão da pesquisa; estabelecimento de critérios para a amostragem; evidenciação dos estudos; avaliação dos estudos; interpretação dos resultados e síntese do conhecimento (Silva et al., 2021).

Os dados foram coletados de julho a agosto de 2021, e foi utilizado o acrônimo PICO: P (População), I (Interesse) e Co (Contexto) para a busca de artigos nas bases de dados (Cardoso et al., 2019). Ficando determinado como P: contribuições do enfermeiro, I: Educação e gerenciamento do cuidado e Co: Cliente nefropata durante tratamento conservador. 
A questão norteadora da revisão integrativa foi: "Quais são as evidências científicas sobre as contribuições do enfermeiro na educação e gerenciamento do cuidado em clientes nefropatas durante o tratamento conservador?"

Foram definidos, para a busca dos dados, os Descritores em Ciências da Saúde (DeCS): "Cuidados de enfermagem", "Tratamento Conservador", "Enfermagem", "Insuficiência Renal Crônica” e "Educação em Saúde”. Da mesma forma, os descritores do Medical Subject Headings (MeSH): "Nursing Care", "Conservative Treatment", "Nursing", "Renal Insufficiency, Chronic" e "Health Education". Por fim, os tesauros EMTREE da EMBASE: "Nursing Care", "Conservative Treatment", "Chronic Kidney Failure" e "Health Education". A combinação dos descritores foi realizada com a utilização do operador booleano $A N D$ e $O R$.

As bases virtuais utilizadas foram: EMBASE (Elsevier), Literatura Latino-Americano e do Caribe em Ciências da Saúde (LILACS), Medical Literature Analysis and Retrieval System Online (PubMed/MEDLINE) e Scientific Electronic Library Online (SciELO). Como critérios de inclusão: artigos originais publicados entre os anos de 2011 e 2021, em português, espanhol ou inglês. E critérios de exclusão: foram excluídos artigos de revisão, reflexões, editoriais, resumos de anais, teses, dissertações, TCCs, boletins epidemiológicos, relatórios de gestão, documentos oficiais de programas nacionais e internacionais, livros e estudos que não respondiam à pergunta de pesquisa estabelecida, inicialmente.

A fim de cumprir a terceira etapa da revisão integrativa, foram estabelecidos os dados a serem coletados de cada estudo: nome dos autores, ano de publicação do artigo, país de origem, metodologia de pesquisa, objetivo e conclusão. Em seguida, os estudos incluídos foram submetidos à classificação por nível de evidência (Centre for Evidence-Based Medicine, 2009). Por fim, procedeu-se à síntese do material encontrado e à respectiva discussão. O corte temporal selecionado se deu devido à escassez de publicações recentes acerca da temática selecionada.

Foi utilizado o Preferred Reporting Items for Systematic Reviews and Meta-Analyses (PRISMA) para a apresentação da seleção dos artigos e a composição do corpus da revisão integrativa (Moher et al., 2009).

\section{Resultados}

A amostra final da pesquisa foi composta por nove artigos, após a leitura dos títulos e resumos e aplicação dos critérios de inclusão e exclusão. Os resultados das buscas nas bases de dados estão apresentados no Fluxograma 1. 
Fluxograma 1. Resultado das buscas nas bases de dados. Rio de Janeiro, RJ, Brasil, 2021.

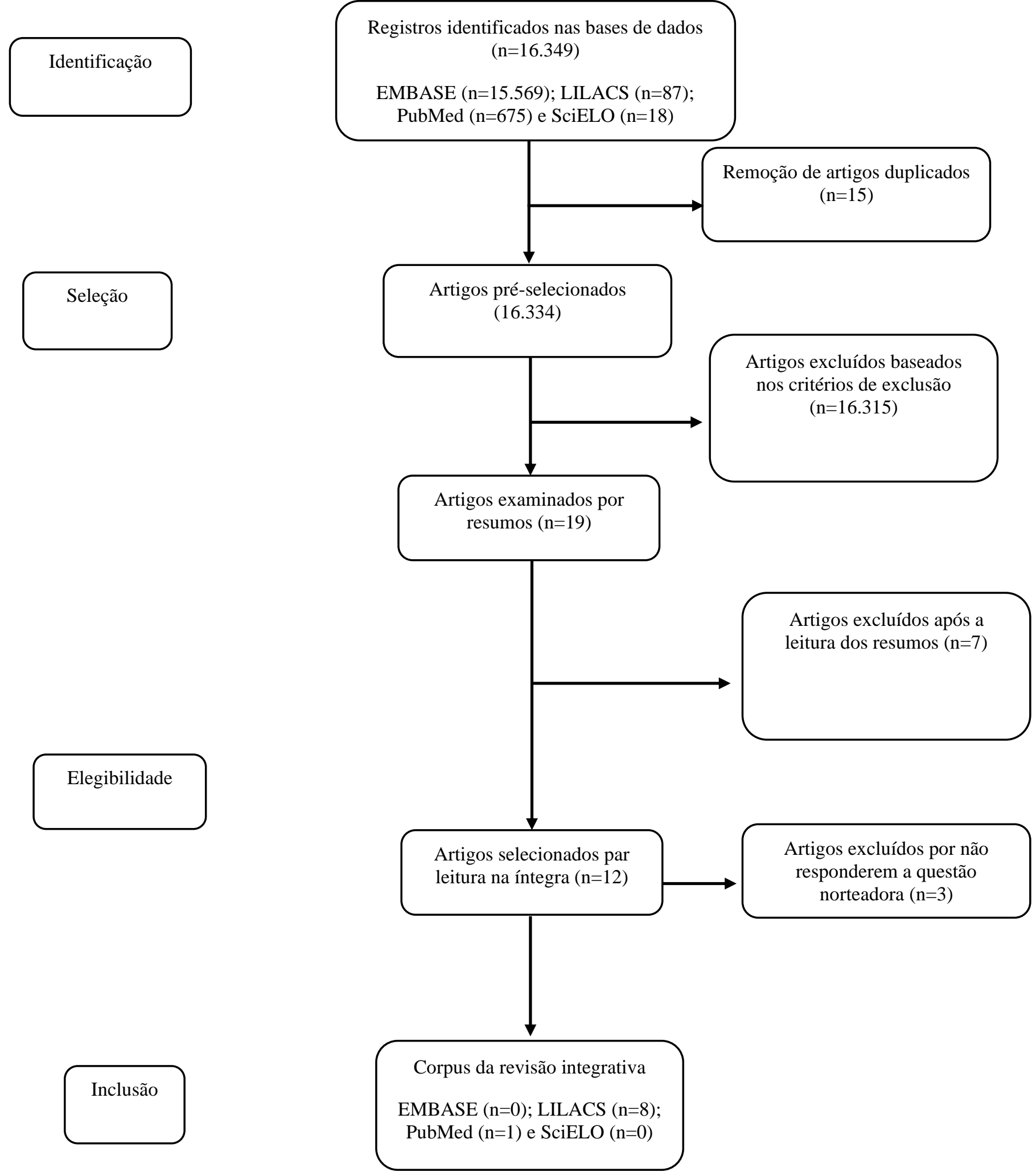

Fonte: Autores (2021).

Os Quadros 1 e 2 apresentam a caracterização dos artigos incluídos na revisão de acordo com os autores, ano de publicação, país de origem, tipo de pesquisa, objetivo e conclusão. 
Quadro 1. Caracterização dos artigos incluídos no estudo de acordo com o nome dos autores, ano de publicação, o país de origem e tipo de pesquisa. Rio de Janeiro, RJ, Brasil, 2021.

\begin{tabular}{|l|c|c|c|c|}
\hline $\mathbf{N}^{\mathbf{0}}$ & Autores & Ano/País & Revista & Método \\
\hline 1 & Menezes et al. & $2020 /$ Brasil & Rev Bras Enferm & Estudo descritivo, documental e metodológico \\
\hline 2 & Silva et al. & $2019 /$ Brasil & Rev Bras Promoç Saúde & Estudo transversal, descritivo e quantitativo \\
\hline 3 & Menezes et al. & $2018 /$ Brasil & Rev Enferm UERJ & Fenomenologia Social \\
\hline 4 & Meira et al. & $2016 /$ Brasil & Rev Rene & Estudo transversal e correlacional \\
\hline 5 & Tam-Tham et al. & $2016 /$ Canadá & Nephrol Dial Transplant & Pesquisa qualitativa \\
\hline 6 & Roso et al. & 2013 a/Brasil & Rev Rene & Pesquisa de campo, descritiva, exploratória e \\
qualitativa
\end{tabular}

Fonte: Autores (2021).

Quadro 2. Caracterização dos artigos incluídos segundo o objetivo e conclusão do estudo. Rio de Janeiro, RJ, Brasil, 2021.

\begin{tabular}{|c|c|c|}
\hline $\mathbf{N}^{\mathbf{o}}$ & Objetivo & Conclusão \\
\hline 1 & $\begin{array}{l}\text { Compreender o significado das ações } \\
\text { educativas na consulta de enfermagem } \\
\text { para clientes renais crônicos em } \\
\text { tratamento conservador e seus familiares. }\end{array}$ & $\begin{array}{l}\text { O estudo permitiu compreender que, no cotidiano desses indivíduos, } \\
\text { eles adquirem habilidade e prática para lidar com suas limitações. As } \\
\text { relações que possuem possibilitam subsídios para o reconhecimento } \\
\text { dos valores não apenas da clínica como do valor enfocado no cuidado } \\
\text { relacional. }\end{array}$ \\
\hline 2 & $\begin{array}{l}\text { Validar quanto ao conteúdo termos da } \\
\text { linguagem especializada de Enfermagem } \\
\text { utilizada no cuidado às pessoas com } \\
\text { doença renal crônica em tratamento } \\
\text { conservador identificados na literatura, } \\
\text { mapeando-os com os termos da } \\
\text { Classificação Internacional para a Prática } \\
\text { de Enfermagem, versão } 2019 \text {, e } \\
\text { representando-os por meio de uma } \\
\text { mandala. }\end{array}$ & $\begin{array}{l}\text { O estudo permitiu validar termos que contribuirão para unificar a } \\
\text { linguagem profissional de enfermagem no cuidado às pessoas com } \\
\text { doença renal crônica. }\end{array}$ \\
\hline 3 & $\begin{array}{l}\text { Avaliar o nível de letramento funcional } \\
\text { em saúde e o conhecimento sobre a } \\
\text { doença renal crônica (DRC) nos pacientes } \\
\text { em tratamento conservador }\end{array}$ & $\begin{array}{l}\text { Os participantes com DRC em tratamento conservador } \\
\text { obtiveram nível inadequado de letramento funcional em saúde e } \\
\text { conhecimento suficiente em relação à doença e ao tratamento. Esses } \\
\text { achados referentes ao conhecimento apontam que apenas a } \\
\text { transferência dele para os usuários não é suficiente para decisões que } \\
\text { envolvem o autogerenciamento da saúde. }\end{array}$ \\
\hline 4 & $\begin{array}{l}\text { Avaliar o nível de fragilidade dos idosos } \\
\text { com doença renal crônica em tratamento } \\
\text { conservador. }\end{array}$ & $\begin{array}{l}\text { Os idosos com doença renal crônica em tratamento conservador } \\
\text { apresentaram algum grau de fragilidade, os maiores níveis foram } \\
\text { correlacionados com menor escolaridade e maior número de } \\
\text { complicações clínicas. }\end{array}$ \\
\hline 5 & $\begin{array}{l}\text { Descrever os aspectos clínicos e sociais } \\
\text { das pessoas com insuficiência renal } \\
\text { crônica em tratamento conservador em um } \\
\text { ambulatório de uremia da região sul do } \\
\text { Brasil }\end{array}$ & $\begin{array}{l}\text { Conclui-se que as alterações laboratoriais estão relacionadas à piora da } \\
\text { função renal, agravamento das doenças cardiovasculares e aumento da } \\
\text { morbidade e mortalidade. O tratamento conservador reduz o ritmo de } \\
\text { progressão da doença, mantendo a função renal e melhorando as } \\
\text { condições clínicas, psicológicas e sociais das pessoas. O enfermeiro } \\
\text { pode desenvolver atividades de educação em saúde efetivas na } \\
\text { promoção da saúde dessas pessoas. }\end{array}$ \\
\hline
\end{tabular}




\begin{tabular}{|l|l|l|}
\hline 6 & $\begin{array}{l}\text { Objetivou-se descrever como pessoas com } \\
\text { insuficiência renal crônica, em tratamento } \\
\text { conservador, cuidam de si }\end{array}$ & $\begin{array}{l}\text { Conclui-se que o cuidado de si dessas pessoas em tratamento } \\
\text { conservador é expresso por atitudes que vão da renúncia à aceitação da } \\
\text { situação de cronicidade. Compreende-se que é } \\
\text { preciso pensar no sentido mais amplo da promoção da saúde, buscando } \\
\text { a qualidade de vida das pessoas em tratamento conservador } \\
\text { da insuficiência renal crônica para o cuidado de si. }\end{array}$ \\
\hline 7 & $\begin{array}{l}\text { Objetivou descrever o cuidado de idosos e e } \\
\text { a participação dos familiares no } \\
\text { tratamento conservador da nefropatia } \\
\text { diabética }\end{array}$ & $\begin{array}{l}\text { Conclui-se que os idosos com nefropatia diabética em tratamento } \\
\text { conservador e seus familiares vivenciam o cuidado permeado por } \\
\text { dificuldades. Assim, necessitam de ajuda profissional para terem } \\
\text { conhecimento, compreenderem e aderirem a este tratamento }\end{array}$ \\
\hline $\begin{array}{l}\text { Objetivou avaliar a adesão dos indivíduos } \\
\text { a este tratamento analisando os fatores } \\
\text { intervenientes para esta }\end{array}$ & $\begin{array}{l}\text { Neste estudo, constatou-se que a maioria dos entrevistados apresenta } \\
\text { baixo nível socioeconômico e grande dificuldade em realizar a dieta e } \\
\text { terapêutica medicamentosa, havendo necessidade real de reformular o } \\
\text { atendimento destes indivíduos promovendo maior abordagem } \\
\text { interdisciplinar incluindo atividades educativas. }\end{array}$ \\
\hline 9 & $\begin{array}{l}\text { Descrever barreiras, facilitadores e } \\
\text { estratégias para melhorar os cuidados } \\
\text { conservadores, não dialíticos e de DRC } \\
\text { pelos MAP na comunidade. }\end{array}$ & $\begin{array}{l}\text { Os profissionais de atenção primária identificaram barreiras e } \\
\text { facilitadores importantes para o tratamento conservador para seus } \\
\text { pacientes mais velhos com DRC no Estágio 5. É necessária uma } \\
\text { investigação mais aprofundada das estratégias potenciais que abordam } \\
\text { as barreiras e capacitam os facilitadores para melhorar a qualidade do } \\
\text { atendimento conservador para idosos na comunidade. }\end{array}$ \\
\hline
\end{tabular}

Fonte: Autores (2021).

O Ano de 2013 foi o que apresentou maior número de publicações com três artigos 33\% (Roso et al., 2013a; Roso et al., 2013b; Jacobi et al., 2013), dos nove artigos incluídos 11\% foi publicado em periódico fora da área de conhecimento da enfermagem, tendo como autores os enfermeiros excetuando o estudo de Tam-Tham et al. (2016). Quanto ao país de origem, destaca-se o Brasil com oito artigos publicados 89\% (Menezes et al., 2020; Silva et al., 2019; Menezes et al., 2018; Meira et al., 2016; Roso et al., 2013 a; Roso et al., 2013b; Jacobi et al., 2013; Medeiros \& Sá, 2011), a Revista RENE foi a com maior número de artigos publicados, totalizando quatro artigos 44\% (Meira et al., 2016; Roso et al., 2013 a; Jacobi et al., 2013; Medeiros \& Sá, 2011), um artigo apenas teve como método a pesquisa quantitativa 11\% (Silva et al., 2019), todos os estudos foram classificados como nível VI que é caracterizado como evidência derivada de um único estudo descritivo ou qualitativo, o que representa baixo nível de evidências (Centre for Evidence-Based Medicine, 2009). O corte temporal, inicialmente, seria de publicações dos últimos cinco anos, dado a escassez de material o corte foi ampliado para os últimos dez anos para maior consistência do estudo.

\section{Discussão}

O ano de 2013 teve maior quantitativo de artigos publicados. Em relação a esse fato, é possível inferir que foi o ano pré-publicação das Diretrizes clínicas para o cuidado ao paciente com doença renal crônica - DRC no sistema único de saúde, onde o tratamento conservador em nefrologia é abordado de forma ampla, desde explicações acerca do que se trata, ao momento de entrada e de sua manutenção, tendo como base o guideline da National Kidney Foundation (KDOQI) de 2022 (Ministério da Saúde 2014a).

O fato de $89 \%$ dos artigos terem sido publicados em periódicos de enfermagem, deve-se ao fato da marcante presença de forma imprescindível dessa categoria nos ambulatórios de tratamento conservador. Pois, é nesse momento que se planeja a individualização da assistência onde inclui a adesão ao autocuidado, a disponibilidade de portas abertas para esclarecimentos, conhecimento das terapias com visitas guiadas aos setores, visando o estimular e promover a adesão do indivíduo ao tratamento (Lins et al., 2017).

O Brasil, como país que mais publicou em tratamento conservador, tendo como base o objetivo e questão norteadora deste estudo se dá ao fato, das diferenças culturais entre os países mais evoluídos que encaram o tratamento conservador de 
forma diferente do país, que é priorizado o retardo do paciente em alguma terapia renal substitutiva, (Metzger et al., 2021; Saaed et al., 2020; Tam-Tham et al., 2016; Teruel et al., 2015).

A revista RENE que teve suas origens na Rede de Enfermagem do Nordeste, teve mais artigos publicados, dado que no estudo de Sarmento et al., (2018) demonstra que o Nordeste brasileiro está um pouco acima da média do país que é de 38\%, quando o assunto é tratamento em nefrologia e aponta também que a DRC nessa região é subnotificada, o que pode explicar a ativa participação do Nordeste na publicação em prol do tratamento conservador.

Haja vista que os estudos qualitativos predominaram por ser um processo analítico para nortear pesquisadores, permitindo atividade reflexiva, mantendo conexão com um todo através da interpretação textual, enquanto os estudos quantitativos fornecem considerável reforço as conclusões obtidas através de dados estatísticos (Gil, 2008).

Considerando que todos os estudos apresentaram nível de evidência $\mathrm{V}$, o mais baixo na hierarquia (Centre for Evidence-Based Medicine, 2009), nos faz refletir o quanto ainda é preciso pesquisar, discutir e produzir conhecimento com maior consistência e fator de impacto para embasar as ações e fazer do tratamento conservador uma ferramenta que possa ser aplicado em todo ambulatório de nefrologia, o que impactará, diretamente, a vida do usuário.

Sendo assim, a atuação do enfermeiro é prevista pela lei n. 7.498 de 1986 como parte integrante da equipe de saúde participando no planejamento, execução, avaliação, elaboração de planos assistenciais de saúde, bem como profissional prescritor de cuidados mediante as consultas de enfermagem. (Ministério da Saúde, 1986).

Além disso, para contextualizar a HAS e a DM, que possuem etiologias diversas, mas que se não tratadas, adequadamente, previamente, preferencialmente, na atenção básica deixarão os clientes suscetíveis e propensos a DRC, por isso, é fundamental a consulta de enfermagem que conhecerá hábitos, padrões alimentares, rede social de apoio e identificar fatores que contribuam para o controle metabólico dos clientes (Ministério da Saúde, 2014b; Neves et al., 2020).

Em outras palavras, a consulta de enfermagem é fator importante para o tratamento do cliente com acometimento renal crônico, através da educação, fazendo com que o paciente entenda o curso de sua doença, a possibilidade de mudança de hábitos de vida, retardo na progressão da doença e no autocuidado (Menezes et al., 2020; Silva et al., 2019; Menezes et al., 2018; Meira et al., 2016; Tam-Tham et al., 2016; Roso et al., 2013a; Roso et al., 2013b; Jacobi et al., 2013; Medeiros \& Sá, 2011).

A realização da consulta de enfermagem é uma estratégia eficaz na detecção precoce de riscos e por ser atividade privativa do enfermeiro, deve-se seguir a sistematização da assistência de enfermagem (SAE), devendo-se "identificar os problemas de saúde-doença, executar e avaliar os cuidados que contribuirão para a promoção, proteção, recuperação e reabilitação da saúde" (Junior et al., 2019, p. 1158).

Em vista disso, a consulta serve como meio de estreitar laços entre os clientes, familiares e a comunidade, gerando confiança, humanização, um olhar holístico, respeito, atenção fazendo com que esses usuários se sintam acolhidos no processo saúde doença. Para tanto, em 2001, o Ministério da Saúde, lançou o programa HIPERDIA com a finalidade de abordagem em conjunto dessas duas morbidades com atendimento multidisciplinar (Ministério da Saúde, 2001).

Por certo, a consulta de enfermagem é um grande agregador de valor no que tange a educação em saúde, pois com os dados obtidos dos pacientes em consultas prévias, o profissional terá subsídios para planejar e executar sua assistência, sendo muito relevante que haja participação de acompanhantes, familiares, amigos ou redes de apoio, para que esse cliente se sinta ativo, ator principal, ganhe consciência de sua condição de saúde e, assim, desenvolvendo grande autonomia e, por fim, permitindo ao profissional de enfermagem garantir satisfatória educação em saúde (Gomes et al., 2021; Menezes et al., 2020; Silva et al., 2019; Menezes et al., 2018; Meira et al., 2016; Tam-Tham et al., 2016; Roso et al., 2013a; Roso et al., 2013b; Jacobi et al., 2013; Medeiros \& Sá, 2011). 
Segundo Medeiros e Sá (2011), a maioria dos pacientes afirmam não sentirem necessidade de palestras educativas, porém acreditam ser de grande importância, pois têm como potencial esclarecer dúvidas, minimizando as dificuldades que surgem durante o tratamento. Com isso, pode ser uma oportunidade para a introdução, não somente de palestras, mas também de vídeos e atividades educativas na sala de espera.

Contudo, é importante saber o quanto tais informações estão sendo aplicadas para melhorar a saúde dessa população em nosso meio. Lima et al. (2014), descrevem que ao utilizar uma intervenção multidisciplinar, atua-se nos fatores de risco para DRC e a análise de desfechos após 1,5 ano de seguimento médio de pacientes atendidos. Entretanto, a necessidade relatada de mais informação, na qual enfatiza-se a dicotomia entre as informações prestadas pelos pacientes que realizam o tratamento dialítico e a necessidade de conhecimento para manutenção do cuidado, torna-se incipiente.

Portanto, segundo Ribeiro e Andrade (2018), acreditam que em relação à Enfermagem, a educação em saúde se constitui como um instrumento fundamental uma vez que, ao subsidiar a assistência eficaz e, por estar relacionada com o autocuidado, tem como objetivo proporcionar o bem-estar e a qualidade de vida diante de qualquer patologia.

Em conformidade, conviver com clientes acometidos com doenças crônicas e a proteção oferecida pelos membros da família, que demonstram compreensão, empatia e estímulo às experiências positivas, são fatores que dignificam a vida dos idosos, principalmente, aqueles portadores de DCNT. Onde, o apoio familiar e de amigos é tido como trocas interpessoais, emocionalmente, favoráveis e adequado conferindo maior segurança (Figueiredo, Ceccon \&Figueiredo, 2021).

No caso de os pacientes desfrutarem do convívio com familiares também é um aspecto positivo, uma vez que a DRC tem como característica progredir com complicações físicas, sociais e emocionais, além de incapacidades, demandando cuidados (Luciano et al., 2012).

Em suma, quando o atendimento é centrado no modelo biomédico, podemos inferir que o tratamento conservador em nefrologia é uma poderosa ferramenta que fará com que esse cliente retarde sua entrada em algum tipo de TRS, pois será acompanhado de forma multidisciplinar, modificando hábitos de vida, sociais, de lazer, nutricionais, psicológicos (Tam-Tham et al., 2016; Roso et al., 2013b).

Contudo, estudo realizado com enfermeiros acerca das várias concepções de promoção da saúde e a interface da sua atuação no ambiente de trabalho, Gurgel et al. (2011), perceberam que tais concepções influenciam, significativamente, nas práticas de cuidado efetivada pelos profissionais. Percebidas tanto no modelo biomédico de prevenção das doenças, quanto pela promoção da saúde, ampliando a qualidade de vida, mudanças de hábitos e novas informações.

Quando abordado a mudança de hábitos com clientes nefropatas, inclui-se adesão nutricional, o abandono do tabagismo, do etilismo, e entender do que se trata a DRC e quais os percursos que possa ser percorrido para que entenda os riscos da doença e benefícios da adesão medicamentosa, pois não são fatores isolados que trarão benefícios, redução da progressão da doença (Roso et al., 2013b).

Afinal, ter uma doença crônica envolve mudanças de hábitos de vida que estão relacionadas à dieta e às atividades físicas, atividades cotidianas, atividades sociais, ao uso de medicações e, em casos, chegando à dependência de pessoas para adaptar-se a um novo modo de viver e lidar com o dia a dia (Vignoto, Freitas, \& Schumacher, 2020).

Para Lima et al. (2014) explicam que é essencial ressaltar que as ações sociais fazem parte de um compromisso social ético, solidário, despojado de qualquer interesse unilateral, tendo como objetivos a promoção e recuperação da saúde, reintegração da pessoa no seu meio social e o desenvolvimento do potencial criativo do profissional na busca pela adesão ao tratamento e adequação a nova realidade.

Entretanto, alguns desses clientes, mesmo após o diagnóstico de DRC e ter que conviver com assiduidade do ambulatório de tratamento conservador, pode não existir mudanças significativas em suas rotinas, para outros é difícil mudar 
antigos hábitos como parar cessação do fumo e/ou da ingestão de álcool, interromper suas atividades, alterar hábitos alimentares (Vignoto, Freitas, \& Schumacher, 2020).

Portanto, nunca é tarde para que a equipe de enfermagem incentive os familiares e pacientes a engajarem no novo estilo de vida, deve-se apoiar o processo de autocuidado tem o papel de auxiliar na identificação e na reflexão sobre as dificuldades e potencialidades para realizar modificações no estilo de vida (Ministério da Saúde, 2014b).

Contudo, a DRC, como o próprio nome já diz, é uma doença incurável e de longa duração, ocasionando limitações ao paciente e repercutindo, assim, na vida deste e de seus familiares. Por isso, a convivência diária com a doença é um processo difícil e doloroso. Essas limitações atreladas, muitas vezes, à negação ao tratamento acarretam quadro de depressão em muitos casos, além de demais complicações, culminado com a vulnerabilidade do paciente (Horta \& Lopes, 2017).

A falta de informação e o desconhecimento sobre a doença e seu tratamento, seja ele conservador ou TRS acabam por reduzir a adesão do paciente ao tratamento e provocando seu estresse. Dessa forma, é de competência do Enfermeiro a explicação não só ao paciente, mas também aos seus familiares sobre a patologia e as consequências da não adesão ao tratamento (Madeiro et al., 2010).

Para que haja a adesão do paciente ao tratamento, é necessária a realização do acolhimento por parte dos profissionais da equipe interdisciplinar, desenvolvendo, assim, de forma gradual, aproximação e parceria em uma relação de confiança entre paciente e equipe de saúde, possibilitando a abertura para o diálogo (Silveira \& Ribeiro, 2005).

Uma equipe interdisciplinar compõe-se por profissionais de diferentes áreas que desenvolvem um projeto específico (Rezende, 2008). O trabalho interdisciplinar é realizado em conjunto através de bases disciplinares específicas na busca de soluções para os problemas de saúde do indivíduo, reconhecendo sua complexidade através de um olhar amplo e múltiplo (Saupe et al., 2005).

Dentro dessa equipe voltada ao paciente com nefropatia, a atuação do enfermeiro é fundamental e de destaque pela sua capacidade de captar e compreender a essência de cada indivíduo, proporcionar suporte emocional e esclarecimento em relação à patologia e orientar sobre as alternativas disponíveis para manter sua qualidade de vida, baseadas individualmente e integralmente ao paciente de forma parceira (Maldaner et al., 2008). E é, justamente, dentro dessa relação enfermeiro paciente onde se faz a educação em saúde. A educação em saúde é justamente toda a orientação baseada no indivíduo, realizada pelo enfermeiro.

A grande justificativa para a eleição do enfermeiro como primordial para a realização da educação em saúde, é pelo fato deste ser o profissional mais presente durante o tratamento do paciente. Sua participação em todas as etapas vividas por este paciente permite conhecer, avaliar, atuar nos cuidados, intervir e preparar ações educativas para proporcionar melhor qualidade de vida o mais, precocemente, possível com a finalidade de redução a um mínimo de complicações, colocando o paciente em posição de autonomia para seu autocuidado e proporcionando seu bem-estar (Horta \& Lopes, 2017).

Vale sempre destacar a importância da Consulta de Enfermagem, momento no qual o enfermeiro conhece e cria vínculo com o paciente, além de desenvolver a educação em saúde. Segundo a Resolução COFEN - 159/93 é uma atividade privativa do enfermeiro, que faz uso de métodos científicos para identificar situações de saúde/doença e necessidades do indivíduo, desenvolver estratégias, prescrever e implementar medidas de enfermagem com o objetivo de promoção, proteção, recuperação ou reabilitação do paciente (Conselho Federal de Enfermagem, 1993). Dessa forma, o enfermeiro realiza Consulta de Enfermagem durante um atendimento ambulatorial em nefrologia, por exemplo, fazendo Educação em Saúde ao paciente.

Em um estudo de Santos et. al (2011), foram destacadas ações em Educação em Saúde, realizadas pelo enfermeiro aos pacientes em tratamento conservador ou TRS, sendo direcionadas sempre ao desenvolvimento da capacidade de autocuidado, sendo elas: orientação sobre o funcionamento da hemodiálise e sobre as medicações utilizadas tanto para o paciente quanto para seus familiares, educação alimentar com orientações gerais sobre alimentos prejudiciais, como os ricos em fósforo 
(miúdos, chocolate, refrigerante), além de enfatizar que café, chá, sopa, sorvete, água de coco, frutas e legumes com muita água (tais como melancia, abacaxi, laranja, tomate, alface) devem ser incluídos no volume total de líquidos ingeridos, encaminhando esse paciente ao Nutricionista para avaliação especializada, orientações sobre cuidados com o cateter de HD e orientações quanto a importância de atividade física e/ou de lazer/recreação para melhoria do bem-estar.

Contudo, a importância da implementação de novas propostas educativas que, ao surgirem do cotidiano, perpassam as técnicas burocráticas e ao assistencialismo vinculado às atuais práticas profissionais. Nesse sentido, o tratamento conservador pode ser considerado uma opção para pacientes que escolham não serem mantidos em TRS devendo ser criado um programa de suporte para esses casos (Santos et al., 2011).

Dessa forma, fica clara a importância da Educação em Saúde ao paciente com nefropatia, para que se torne entendedor de sua doença e consiga se adequar às limitações ocasionadas por ela, e pratique com tranquilidade seu autocuidado seguindo o tratamento, adequadamente, além de criar vínculo de confiança não só com o enfermeiro, mas com toda a equipe interdisciplinar.

E durante as buscas realizadas nas bases de dados, emergiram artigos que abordavam o tratamento conservador, mas não da forma realizada no Brasil e, sim, como um método utilizado para os pacientes que decidiam sair das TRS e ingressar no tratamento conservador ou como trabalhado no exterior, cuidados paliativos.

Ao entrar em tratamento conservador nos moldes de cuidado paliativo, o paciente deve estar em estágio 5 da classificação da DRC, onde sua TFG $<15 \mathrm{ml} / \mathrm{min} / 1,73 \mathrm{~m}^{2}$ e é visto como mais uma opção de tratamento e uma abordagem que melhora a qualidade de vida dos pacientes e suas famílias (Kamar, Tam-Tham, \& Thomas 2017).

De acordo com o mencionado anteriormente, no Brasil (2014a), o paciente em estágio 5 deve ser acompanhado por equipe multiprofissional e com tratamento nos fatores modificáveis para a progressão da DRC, e não tratado como cuidados paliativos de fim de vida e que, no geral, a maioria das mortes ocorreu no local de escolha, e com qualidade (Kamar, TamTham, \& Thomas 2017; Susanto et al., 2018).

Conquanto, a maioria dos profissionais se concentrava em tratamentos ativos, obscurecendo a natureza terminal da DRC. "As barreiras significativas no nível do médico para discutir o manejo conservador incluíram o medo de suspender o tratamento benéfico, a incerteza do prognóstico e a relutância em perturbar os pacientes. A trepidação sobre as respostas emocionais dos pacientes impediu as discussões sobre os objetivos de cuidado e qualidade de vida" (Ladin et al, 2018, p. 629).

\section{Conclusão}

A abordagem dos estudos acerca das contribuições do enfermeiro na educação e o gerenciamento do cuidado ao paciente nefropata em tratamento conservador ainda é incipiente, pois poucos apresentam discussões robustas sobre. É necessário estimular a participação das pessoas no seu tratamento, tendo em vista que a melhora na qualidade de vida pode reduzir, significativamente, a progressão da doença.

Destaca-se o papel fundamental do enfermeiro inserido nos ambulatórios de tratamento conservador, sendo que articula com as demais categorias profissionais em busca de melhores condições e qualidade. Evidenciou-se a necessidade de conhecer a visão do paciente em relação a doença renal crônica, com o intuito de partilhar melhor, as decisões a serem tomadas acerca de tratamento, rede de apoio e profissionais de saúde.

Este estudo apresentou limitações caracterizadas pela especificidade do tema e por ser em uma realidade nacional, com poucos ambulatórios de tratamento conservador que possuem equipe multidisciplinar. Como contribuições, aponta a necessidade de se vislumbrar a atenção oferecida às pessoas com DRC, sendo que a pesquisa possibilita reflexões que poderão gerar mudanças no campo prático. 
Sendo assim, sugere-se a realização de novos estudos futuros com a temática principal do estudo e principalmente com relação ao tratamento conservador como método de cuidados paliativos, com vista a qualidade de vida dos pacientes nefropatas; a inclusão da temática na formação do profissional enfermeiro e que contudo revela a necessidade do aprofundamento nos conteúdos de nefrologia.

\section{Referências}

Abreu, P. F. \& Moura, L. R. R. (2018). Epidemiologia da doença renal crônica. In L. R. R. Moura, M. A. R. Alves, D. R. Santos \& R. P. Filho (Ed.), Tratado de Nefrologia, 911. Atheneu.

Bardin, L. (2011). Análise de conteúdo. Almedina.

Bica, M. C., Cremonese, L., Barreto, C. M. Rodrigues, A. L. M. \& Alves, F. Q. (2020). Gerenciamento do cuidado em estratégias saúde da família na percepção de enfermeiros. Rev Enferm UFSM, 10(e74), 1-18. https://doi.org/10.5902/2179769242518.

Cardoso, V., Trevisan, I., Cicolella, D. A., \& Waterkemper, R. (2019). Revisão sistemática de métodos mistos: método de pesquisa para a incorporação de evidências na enfermagem. Texto \& Contexto - Enfermagem, 28(e20170279), 1-12. https://dx.doi.org/10.1590/1980-265X-TCE-2017-0279.

Castro, M. C. M. (2019). Tratamento conservador de paciente com doença renal crônica que renuncia à diálise. Braz $J$ Nephrol, 41(1), 95-102. https://dx.doi.org/10.1590/2175-8239-JBN-2018-0028.

Centre for Evidence-Based Medicine. (2009). Oxford Centre for Evidence-Based Medicine: levels of evidence (2009). CEBM. https://www.cebm.ox.ac.uk/resources/levels-of-evidence/oxford-centre-for-evidence-based-medicine-levels-of-evidence-march-2009.

Conselho Federal de Enfermagem. Resolução COFEN n. 159, de 19 de abril de 1993. Dispõe sobre a consulta de enfermagem. http://www.cofen.gov.br/ resoluocofen1591993_4241.html.

Figueiredo, A. E. B., Ceccon, R. F., \& Figueiredo, J. H. C. (2021). Doenças crônicas não transmissíveis e suas implicações na vida de idosos dependentes. Ciên. Saúde Colet, 26(01), 77-88. https://doi.org/10.1590/1413-81232020261.33882020.

Gil, A. C. (2008). Métodos e técnicas de pesquisa social. Atlas.

Gomes, A. F., Yoshimura, B. K., Souza, H. F., Lima, N. R., Paula, S. A., \& Marques, M. V. (2021). A importância da consulta de enfermagem cardiológica na prevenção de doenças e promoção de saúde. Saúde Coletiva, 11(63), 5466-5475. https://doi.org/10.36489/saudecoletiva.2021v11i63p5466-5475.

Gurgel, M. G. I., Alves, M. D. S., Moura, E. R. F., Pinheiro, P. N. C., Rêgo, R. M. V., \& Passos, M. L. L. (2011). Promoção da saúde no contexto da estratégia saúde da família: concepções e práticas da enfermeira. Esc. Anna Nery, 15(3), 610-615. https://doi.org/10.1590/S1414-81452011000300024.

Horta, H. H. L., \& Lopes, M. L. (2017). Complicações decorrentes do tratamento dialítico: contribuição do enfermeiro no cuidado e educação ao paciente. Revista Enfermagem Contemporânea, (6)2, 221-227. https://doi.org/10.17267/2317-3378rec.v6i2.1457.

Jacobi, C. S., Beuter, M., Maldaner, C. N., Roso, C. C., Pauletto, M. R., \& Girardon-Perlini, N. M. O. (2013). O cuidado de idosos com nefropatia diabética em tratamento conservador. Rev Rene, 14(4), 765-773. http://www.periodicos.ufc.br/rene/article/view/3541/2781.

Júnior, A. R. C., Abreu, L. D. P., Araújo, A. F., Torres, R. A. M., \& Silva, M. R. F. (2019). Consulta de enfermagem no cuidado ambulatorial às juventudes. Rev Enferm UFPE online, 13(4), 1157-1166. https://doi.org/10.5205/1981-8963-v13i04a239115p1157-1166-2019.

Kamar, F. B., Tam-Tham, H., \& Thomas, C. (2017). A Description of Advanced Chronic Kidney Disease Patients in a Major Urban Center Receiving Conservative Care. Canadian Journal of Kidney Health and Disease, 4, 1-7. https://doi.org/10.1177/2054358117718538.

Ladin, K., Pandya, R., Kannam, A., Loke, R., Oskoui, T., D Perrone, R., Meyer, K. B., Weiner, D. E., \& Wong, J. B. (2018). Discussing Conservative Management With Older Patients With CKD: An Interview Study of Nephrologists. Am J Kidney Dis., 71(5), 627-635. https://doi.org/10.1053/j.ajkd.2017.11.011.

Lima, M. A., Sousa, G. R., Sousa, A. M., Felipe, G. F., Oliveira, A. S. S. \& Formiga, L. M. F. (2014). Educação em saúde para pacientes em hemodiálise. Rev Enferm UFPE online, 8(6), 1510-1515. https://doi.org/10.5205/1981-8963-v8i6a9839p1510-1515-2014.

Lins, S. M. S. B., Tavares, J. M. A. B., Silva, F. V. C., Campos, T. S., Lima, V. K., Broca, P., Rocha, R. G, \& Pereira E. P. (2017, junho ). Implantação da consulta de enfermagem no ambulatório de atendimento ao paciente renal em tratamento conservador. Anais do primeiro encontro internacional do processo de enfermagem: o raciocínio clínico de enfermagem e a era digital, Campinas, SP, Brasil, 1. https://proceedings.science/enipe/papers/implantacao-da-consulta-de-enfermagem-no-ambulatorio-de-atendimento-ao-paciente-renal-em-tratamentoconservador-.

Luciano, E. P., Luconi, P. S., Sesso, R. C., Melaragno, C. S., Abreu, P. F., Reis, S. F. S., Furtado, R. M. S., \& Ruivo, G. F. (2012). Estudo prospectivo de 2151 pacientes com doença renal crônica em tratamento conservador com abordagem multidisciplinar no Vale do Paraíba, SP. Braz J Nephrol, 34(3), 226-234. https://doi.org/10.5935/0101-2800.20120003.

Madeiro, A. C., Machado, P. D. L. C., Bonfim, I. M., Braqueais, A. R., \& Lima F. E. T. Adesão de portadores de insuficiência renal crônica ao tratamento de hemodiálise. Acta Paul Enferm, 23(4), 546-551. https://doi.org/10.1590/S0103-21002010000400016. 
Maldaner, C. R., Beuter, M., Brondani, C. M., Budo, M. L. D., \& Paulleto, M.R. (2008). Fatores que influenciam a adesão ao tratamento na doença crônica: o doente em terapia hemodialítica. Rev Gaúcha Enferm, 29(4), 647-53. https://seer.ufrgs.br/rgenf/article/download/7638/4693.

Medeiros, M. C. W. C., \& Sá, M. P. C. (2011). Adesão dos portadores de doença renal crônica ao tratamento conservador. Rev Rene, 12(1), 65-72. http://www.periodicos.ufc.br/rene/article/view/4135/3217.

Meira, A. S., Batista, M. A., Pereira, R. M. P., Rodrigues, R. A. P., Fhon, J. R. S., \& Kusumota L. (2016). Fragilidade em idosos com doença renal crônica em tratamento conservador. Rev Rene, 17(3), 386-392. http://dx.doi.org/10.15253/2175-6783.2016000300012.

Menezes, H. F., Rosas, A. M. M. T. F., Camacho, A. C. L. F., Souza, F. S., Rodrigues, B. M. R. D., \& Silva, R. A. R. (2018). Significado das ações educativas na consulta de enfermagem para clientes renais crônicos e familiares. Rev Enferm UERJ, 26, e31921. http://dx.doi.org/10.12957/reuerj.2018.31921.

Menezes, H. F., Camacho, A. C. L. F., Lins, S. M. S. B., Campos, T. S., Lima, F. R., Jales, A. K. F. A., \& Silva, R. A. R. (2020). Termos da linguagem especializada de enfermagem para pacientes renais crônicos em tratamento conservador. Rev Bras Enferm, 73(Suppl6), e20190820. http://dx.doi.org/10.1590/0034-7167-2019-0820.

Metzger, M., Yoder, J., Fitzgibbon, K., Blackhall, L., \& Abdel-Rahman, E. (2021). Nephrology and Palliative Care Collaboration in the Care of Patients With Advanced Kidney Disease: Results of a Clinician Survey. Kidney Med, 3(3), 368-377. https://doi.org/10.1016/j.xkme.2021.01.008.

Ministério da Saúde. (Brasil). (1986). Lei n. 7.498, de 25 de junho de 1986. Dispõe sobre a regulamentação do exercício da enfermagem, e dá outras providências. http://www.planalto.gov.br/ccivil_03/leis/17498.htm.

Ministério da Saúde. (Brasil) (2001). Hipertensão arterial sistêmica (HAS) e Diabetes mellitus (DM): protocolo. Brasília: Ministério da Saúde. https://bvsms.saude.gov.br/bvs/publicacoes/cd05_06.pdf.

Ministério da Saúde. (Brasil). (2020). Plano de ações estratégicas para o enfrentamento das doenças crônicas e agravos não transmissíveis no Brasil. https://antigo.saude.gov.br/images/pdf/2020/October/01/Plano-DANT-vers--o-Consulta-p--blica.pdf.

Ministério da Saúde. (Brasil). (2014a). Diretrizes clínicas para o cuidado ao paciente com doença renal crônica - DRC no sistema único de saúde. https://portalarquivos2.saude.gov.br/images/pdf/2014/marco/24/diretriz-cl--nica-drc-versao-final.pdf.

Ministério da Saúde. (Brasil). (2014b). Estratégias para o cuidado da pessoa com doença crônica. Ministério da Saúde. https://bvsms.saude.gov.br/bvs/publicacoes/estrategias_cuidado_pessoa_doenca_cronica_cab35.pdf.

Moher, D., Liberati, A., Tetzlaff, J., Altman, D. G., \&The PRISMA Group. (2009). Preferred Reporting Items for Systematic Reviews and Meta-Analyses: The PRISMA Statement. Plos Medicine, 6(7), e100097. https://doi.org/10.1371/journal.pmed.1000097.

Neves, P. D. M. M., Sesso, R. C. C., Thomé, F. S., Lugon, J. R, \& Nasciment, M. M. (2020). Censo Brasileiro de Diálise: análise de dados da década 20092018. Braz J Nephrol, 42(2), 191-200. https://doi.org/10.1590/2175-8239-JBN-2019-0234.

Rezende, A. D. (2008). Planejamento estratégico para organizações privadas e públicas: guia prático para elaboração do projeto de plano de negócios. Rio de Janeiro: Brasport.

Ribeiro, W. A., Andrade, M., Fassarella, B. P. A., Santana, P. P. C., Costa, P. A. F. S., \& Morais, M. C. (2018). Enfermeiro protagonista na educação em saúde para o autocuidado de pacientes com doenças renal crônica. Revista Pró-univerSUS, 09(2), 60-65. http://editora.universidaded evassouras.edu.br/index.php/RPU/article/view/1378/1037

Roso, C. C., Beuter, M., Bruinsma, J. L., Silva, J. H., Timm, A. M. B. \& Pauletto, M. R. (2013a). Aspectos clínicos das pessoas com insuficiência renal crônica em tratamento conservador. Rev Rene, 14(6), 1201-1208. http://www.periodicos.ufc.br/rene/article/view/3742/2962.

Roso, C. C., Beuter, M., Kruse, M. H. L., Girardon-Pelini, N. M. O., Jacobi, C. S., \& Cordeiro, F. R. (2013b). O cuidado de si de pessoas em tratamento conservador da insuficiência renal crônica. Texto Contexto Enferm, 22(3), 739-745. https://doi.org/10.1590/S0104-07072013000300021.

Saeed, F., Adams, H., \& Epsten, R. M. (2020). Matters of Life and Death: Why Do Older Patients Choose Conservative Management? Am J Nephrol, 51(1), 35-42. https://doi.org/10.1159/000504692.

Sarmento, L. R., Fernandes, P. F. C. B. C., Pontes, M. X., Correia, D. B. S., Chaves, V. C. B, Carvalho, C. F. A., Arnaud, T. L., Santos, M. H. S., Barreto, L. C. B., \& Moliterno, L. A. A. (2018). Prevalência das causas primárias de doença renal crônica terminal (DRCT) validadas clinicamente em uma capital do Nordeste brasileiro. J. Braz Nephrol, 40(2), 130-135. https://doi.org/10.1590/2175-8239-JBN-3781.

Santos, I., Rocha, R.P.F., \& Berardinelli, L.M.M. (2011) Necessidades de orientação de enfermagem para o autocuidado de clientes em terapia de hemodiálise. Rev. Bras. Enferm., 64(2), 335-342. https://doi.org/10.1590/S0034-71672011000200018.

Saupe, R., Cutolo, L. R. A., Wendhausen, A. L. P., \& Benito, G. A. V. (205). Competência dos profissionais da saúde para o trabalho interdisciplinar.

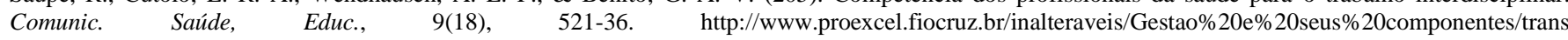
discipl\%20no\%20traba\%20de\%20saude.pdf.

Siewert, J. S., Rodrigues, D. B., de Malfussi, L. B. H., Andrade, S. R. \& Erdmann, A. L. (2017). Gestão do cuidado integral em enfermagem: reflexões sob a perspectiva do pensamento complexo. Reme, 21(e1047), 1-5. http://www.dx.doi.org/10.5935/1415-2762.20170057.

Silva, B. A. B., Pereira, F. A. R., Oliveira, L. F. A., Lara, S. H. O., Bressan, V. R., \& Goyatá, S. L. T. (2021). Contribuições da terapia comunitária integrativa na promoção da saúde: revisão integrativa. R. pesq.: cuid. fundam. Online, 13, 843-848. https://doi.org/10.9789/2175-5361.rpcfo.v13.9238

Silva, J. R. R., Luz, G. O. A., Silva, S. M. B., Medeiros, L. K. A., Junior, J. L. S. \& Santos, I. C. R. V. (2019). Letramento funcional em saúde e o conhecimento dos doentes renais crônicos em tratamento conservador. Rev Bras Promoç Saúde, 32, 9470. https://periodicos.unifor.br/RBPS/article/view/9470. 
Research, Society and Development, v. 10, n. 14, e136101421824, 2021

(CC BY 4.0) | ISSN 2525-3409 | DOI: $\eta \tau \tau \pi: / / \delta \xi . \delta o 1 . o \rho \gamma / 10.33448 / \rho \sigma \delta-\varpi 10 \imath 14.21824$

Silveira, L. M. C., \& Ribeiro, V. M. B. (2005). Grupo de adesão ao tratamento: espaço de "ensinagem" para profissionais de saúde e pacientes. Interface: Comunicação, Saúde, Educação, 9(16), 91- 104. https://doi.org/10.1590/S1414-32832005000100008.

Susanto, C., Kooman, J., Courtens, A. M., \& Konings, C. J. A. M. (2018). Conservative care as a treatment option for patients aged 75 years and older with CKD stage V: a National survey in the Netherlands. European Geriatric Medicine, 9(2), 235-242. https://doi.org/10.1007/s41999-018-0031-9.

Tam-Tham, H., Hemmelgarn, B. R., Campbell, D. J. T., Thomas, C. M., Fruetel, K., Quinn, R. R., \& King-Shier, K. M. (2016). Primary care physicians' perceived barriers, facilitators and strategies to enhance conservative care for older adults with chronic kidney disease: a qualitative descriptive study. Nephrol Dial Transplant, 31(11), 1864-1870. https://doi.org/10.1093/ndt/gfv408.

Tam-Tham, H., King-Shier, K. M., Thomas, C. M., Quinn, R. R., Fruetel, K., Davison, S. N., \& Hemmelgarn, B. N. (2016). Prevalence of Barriers and Facilitators to Enhancing Conservative Kidney Management for Older Adults in the Primary Care Setting. Clin J Am Soc Nephrol, 11(11), 2012-2021. https://doi.org/10.2215/cjn.04510416.

Teruel, J. L., Vion, V. B., Couto, A. G., Gorrín, M. R., Fernández-Lucas, M., Mendiola, N. R., \& Quereda, C. (2015). Elección de tratamiento conservador en la enfermedad renal crónica. Nefrologia, 35(3), 273-279. https://doi.org/10.1016/j.nefro.2015.05.005.

Vignoto, S., Freitas, H. M. R., \& Schumacher, B. (2020). Percepções dos pacientes com insuficiência renal crônica em hemodiálise com relação às mudanças dos hábitos de vida. Redes - Revista Interdisciplinar Do IELUSC, 1(3), 157-168. http://revistaredes.ielusc.br/index.php/revistaredes/article/view/97.

World Health Organization. (2019). Global action plan for the prevention and control of noncommunicable diseases $2013-2020$. http://apps.who.int/iris/bitstream/10665/94384/1/9789241506236_eng.pdf. 